\title{
Adaptive Boundary Conditions for Physically Based Follow-Up Breast MR Image Registration
}

\author{
Liesbet Roose, Dirk Loeckx, Wouter Mollemans, Frederik Maes, \\ and Paul Suetens
}

Katholieke Universiteit Leuven, Faculties of Medicine and Engineering, Medical Image Computing (Radiology - ESAT/PSI), University Hospital Gasthuisberg, Herestraat 49, B-3000 Leuven, Belgium

liesbet.roose@uz.kuleuven.ac.be

\begin{abstract}
This paper presents an algorithm for non-rigid registration of breast MRI follow-up images that compensates for differences in patient positioning while maintaining real anatomical and pathological changes. The proposed method uses a biomechanical model to constrain the deformation of the internal breast tissue according to elastic continuum mechanics, which is driven by suitable boundary conditions that align the breast surfaces in the images to be registered. Typically, such boundary conditions impose one-to-one surface point correspondences that are established a priori. We investigate alternative, more flexible boundary conditions that do not depend on fixed point correspondences and do not assume completely accurate breast surface segmentation in both images. More specifically, we allow for sliding motion of one surface over the other during deformation as well as for restricted motion perpendicular to the initially segmented boundary surface, based on the internal elastic forces and local intensity information. We evaluate the impact of different boundary conditions on registration quality from the subtraction images obtained for repeated scans of healthy volunteers with intermediate repositioning, using rigid body and free form whole volume intensity based registration for comparison, and also present initial results for actual patient data. Our results demonstrate a drastic reduction in subtraction artifacts using our model, without compromising the biomechanical validity of the deformation field such as unrealistically large local volume changes as with traditional voxel intensity based registration.
\end{abstract}

\section{Introduction}

Follow-up breast MRI imaging is used to supervise suspicious lesions or to evaluate the effect of breast cancer therapy by comparing images acquired at different time points. Analysis and comparison of such follow-up images would be facilitated by a proper geometric registration of the images that corrects only for changes in patient positioning, while maintaining diagnostically relevant anatomical and pathological differences. While classical free form methods that incorporate intensity information over the whole image may erroneous remove diagnostically relevant features during the registration process, we adopt 
a biomechanically based registration technique that only allows for physically feasible elastic tissue deformations. Such techniques have been applied successfully for example in brain [1], prostate 3] and liver [4] registration. The organ is therefore represented as a finite element model [5] that deforms under the influence of boundary conditions, typically defined on the organ border.

Obviously, the definition of accurate boundary conditions is a crucial step to obtain a good registration result. Most authors [1]34 establish one-to-one point correspondences before solving the finite element model, e.g. by deformable surface matching [1] or by intensity block matching [2]. Liang et al. 6] iteratively optimize the correspondences to minimize internal energy. The breast is enclosed between the skin surface and the internal muscle surface, but these surfaces lack outstanding details or specific intensity patterns. Moreover, a completely accurate segmentation of these enclosing surfaces is difficult, especially for the muscle surface. Furthermore, muscle and skin do not form a closed surface and have no clearly defined border. Hence, the techniques to define boundary conditions as mentioned before are difficult to apply. Therefore, we propose a coupled system of a biomechanical model and iteratively updated boundary conditions that combines known elastic properties of the tissue with a matching of the enclosing surfaces and the underlying image intensities in their neighborhood.

\section{Method}

Figure 1 presents an overview of our biomechanically based intra-subject multitemporal MR breast image registration, used to register floating image $F$ with reference image $R$ by applying a transformation $\phi: \mathbb{R}^{3} \rightarrow \mathbb{R}^{3}$, such that $F(\phi(\mathbf{x}))$ corresponds to $R$. The core idea of the registration approach presented is (1) the representation of the tissue as a biomechanically based model defined over a volumetric mesh and (2) the application of suitable boundary conditions to match this mesh with the skin and muscle surface of the reference image.

\subsection{Preprocessing}

The construction of the mesh and the determination of the boundary conditions requires the breast and skin surface in the reference image to be identified. To this end, the breast tissue is first segmented out of the MR images by thresholding followed by manual correction [7. Skin and muscle surface of the reference image are converted to triangular surface meshes, while the breast tissue in the floating image is converted to a volumetric mesh. Since the breast tissue has an irregular form, tetrahedral elements are chosen here for their flexibility in representing various geometries. These elements are known to have a poor performance for large strains 8], but deformations remain limited in this application.

\section{$2.2 \quad$ Elastic Model}

To find a biomechanically plausible $\phi$, the deformation of the tissue under certain boundary conditions is computed based on continuum mechanics equations. The 


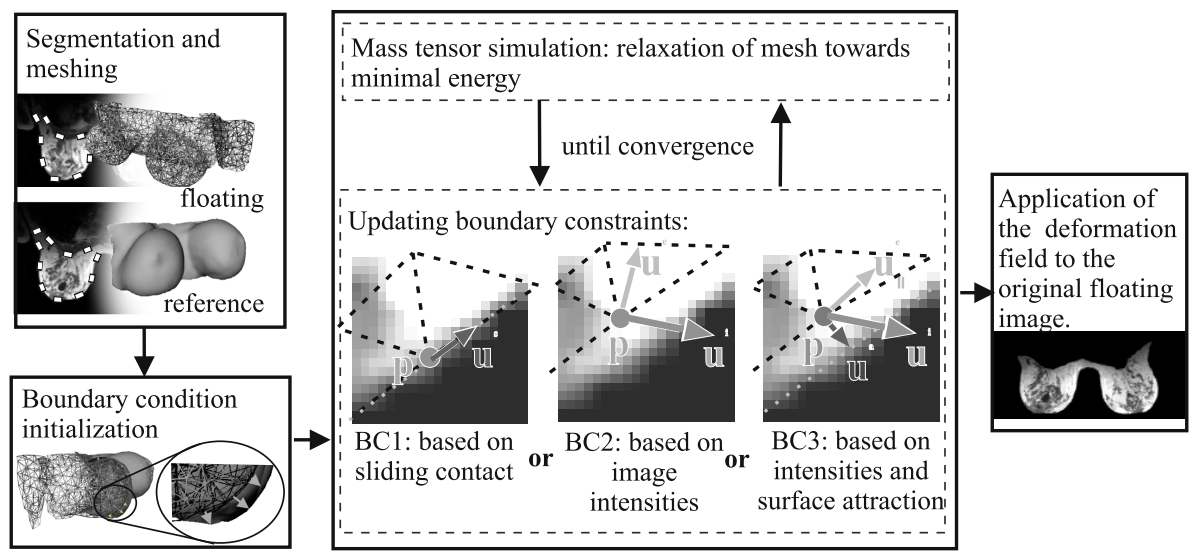

Fig. 1. Overview of the registration approach

solution strategy used in this paper solves the same equations as the classical finite element model based on linear elasticity theory, but uses the mass tensor model (MTM) proposed by Cotin et al. 9] to define the internal forces $\mathbf{f}_{i}$ at each vertex $i$ of the mesh

$$
\mathbf{f}_{i}=\mathbf{K}_{i i} \mathbf{u}_{i}+\sum_{j \in N(i)} \mathbf{K}_{i j} \mathbf{u}_{j}
$$

where $\mathbf{u}$ is the displacement of a vertex relative to its original position, $N(i)$ is the set of neighboring points of point $i$ and the $\mathbf{K}_{i j}$ are tensors determined by the rest position and the material characteristics (Young's modulus $E$ and Poisson coefficient $\nu$ ) of the tetrahedra adjacent to vertex $i$. According to previous research [1011, we approximate the breast tissue as a homogeneous linear elastic material model with $\nu=0.45$ (corresponding to almost incompressibility). Because of the linear equations and the absence of external forces, $E$ needs not to be estimated 12 .

An equilibrium configuration is attained if all forces are minimal: the force in points without boundary conditions (free points) should vanish completely, while the forces in points with a boundary condition can be counteracted partially or completely by the boundary condition. The solution is found in a quasi-static iterative way: in every iteration $t$ the new position $\mathbf{x}_{i}^{t+1}$ of a point $i$ is computed as the position where the force in point $i$ is minimal while all other points remain fixed. For free points, the necessary displacement $\mathbf{u}_{i}^{e}$ is easily computed from (1) as $-\mathbf{K}_{i i}^{-1} \sum_{j \in N(i)} \mathbf{K}_{i j} \mathbf{u}_{j}^{t}$. The equations for points with boundary conditions are discussed in the next section. The equilibrium configuration defines the function values of $\phi$ at the mesh points. These values are linearly interpolated to evaluate $\phi$ over all points inside the mesh. Some boundary conditions considered in section 2.3 also need the deformation field outside the mesh in the neighborhood of the segmented surfaces. Therefore, the deformation field of the tetrahedra at the mesh surfaces is extrapolated to a limited region of interest outside the mesh. 
A continuous extension of $\phi$ is obtained by defining the deformation of a point outside the mesh to be the weighted sum of the deformation of the adjacent tetrahedra, inversely proportional to the distance to the respective tetrahedra.

\subsection{Boundary Conditions}

The deformation of the finite element model is driven by boundary conditions applied to the vertices of both the skin and the muscle surface, in order to align both surfaces in the floating and the reference images. This deformes the second image as if it had been acquired in the position of the first image. Obviously, if the breasts have changed so much at the time of the second acquisition that it would be impossible to deform them into the position of the first image, this approach is meaningless (for example after considerable tissue growth or atrophy).

To initialize the boundary conditions, the segmented surfaces in the floating image are fit rigidly with the surfaces in the reference image. All vertices on a surface in the floating image are projected to the nearest point on the corresponding surface in the reference image and kept fixed during the first 50 iterations. After this initial relaxation, the correspondences are updated. We propose three different approaches, using the knowledge of the segmented surface and/or the underlying image intensities.

BC1: Sliding surface contact. A first scheme assumes a perfect segmentation of the surfaces, and uses the segmented surfaces in the reference image as a guidance: a surface point in the mesh can move under the influence of forces in the model, but is constrained to a sliding motion along the surface in the reference image. This is implemented by splitting the internal force $\mathbf{f}$ on a surface point into the component tangential to the reference surface $\mathbf{f}_{||}$and the perpendicular component $\mathbf{f}_{\perp}$. An optimal displacement $\mathbf{u}^{s}$ is searched as the position with minimal $\mathbf{f}_{||}$, while $\mathbf{f}_{\perp}$ is counteracted by the boundary condition.

BC2: Image intensity guided surface matching. The second scheme is designed to correct segmentation inaccuracies by incorporating extra intensity information in the region of the surfaces. The motion of the surface points is therefore driven by the underlying image intensities, while the elastic model is used as a regularizer. This approach is similar to classical image intensity based registration methods, but is only applied to the surface points. To implement this boundary condition, the displacement $\mathbf{u}$ of a surface point with position $\mathbf{p}$ is a linear combination of the displacement $\mathbf{u}^{e}$ imposed by the elastic model and a displacement $\mathbf{u}^{i}$ that aims at minimizing the local intensity difference $D=\sum_{\mathbf{x}}(F(\mathbf{x})-R(\phi(\mathbf{x})))^{2}$. We define $\mathbf{u}^{i}$ to be proportional to the gradient of $D$ with respect to $\mathbf{p}$ :

$$
\begin{aligned}
\mathbf{u}^{i}=\alpha \frac{1}{N_{x}} \frac{d D}{d \mathbf{p}} & =\frac{\alpha}{N_{x}} \frac{d\left(\sum_{\mathbf{x}}(F(\mathbf{x})-R(\phi(\mathbf{x})))^{2}\right)}{d \mathbf{p}} \\
& =\alpha \frac{-2}{N_{x}} \sum_{\mathbf{x}}(F(\mathbf{x})-R(\phi(\mathbf{x}))) \frac{\partial R(\phi(\mathbf{x}))}{\partial \phi(\mathbf{x})} \frac{d \phi(\mathbf{x})}{d \mathbf{p}}
\end{aligned}
$$


with $\frac{\partial R(\phi(\mathbf{x}))}{\partial \phi(\mathbf{x})}$ the gradient of the reference image, $\frac{d \phi(\mathbf{x})}{d \mathbf{p}}$ the displacement of $\mathbf{x}$ due to a change in $\mathbf{p}, N_{\mathbf{x}}$ the number of voxels and $\alpha$ a weighting parameter. The displacement for a surface point is then computed as the average of $\mathbf{u}^{e}$ and $\mathbf{u}^{i}$ (see Fig. 1):

$$
\mathbf{u}=\frac{\mathbf{u}^{e}+\mathbf{u}^{i}}{2}
$$

BC3: Combined surface attracted and image intensity guided matching. The third scheme is a combination of the previous two: surface points try to align the underlying image intensities, but are regularized by the elastic model and a surface attraction force to keep them in the neighborhood of the segmented surface. This attraction force can be imagined as a spring between the current position of the point and its nearest point on the surface. This regularizes the image intensity driven movement in the direction perpendicular to the surface. Regularization of the movement tangential to the surface is provided by the tangential component of the elastic model $\mathbf{u}_{\|}^{e}$. With $\mathbf{u}^{a}$ the vector between the current position and the nearest surface point (see Fig. 1), the displacement of a surface point is given by

$$
\mathbf{u}=\frac{\left(\mathbf{u}_{\|}^{e}+\mathbf{u}^{a}\right)+\mathbf{u}^{i}}{2} .
$$

\section{Experiments and Results}

Three normal volunteers were scanned twice using a clinical breast MRI imaging protocol $\left(3-\mathrm{D}\right.$ FLASH, TR $=9 \mathrm{~ms}, \mathrm{TE}=4.7 \mathrm{~ms}$, flip angle $=25^{\circ}, \mathrm{FOV}=384 \mathrm{~mm}$ and axial slice orientation), resulting in images of $384 \times 384 \times 64$ voxels with a size of $1.04 \mathrm{~mm} \times 1.04 \mathrm{~mm} \times 2 \mathrm{~mm}$. After the first scan, the subjects were asked to step out of the MR scanner, to stand upright and to reposition themselves in the scanner. Since the two images are acquired immediately after each other, there is no anatomical change between the two images and all image differences are due to different positioning. The clinical data consists of 8 image pairs of different patients.

The segmented breast tissue was meshed with a mean edge length of $10 \mathrm{~mm}$, resulting in meshes with 3800 to 6500 tetrahedra, depending on the breast size. Each couple of images was registered with the three types of boundary conditions to evaluate their ability to compensate for different patient positioning and their compliance to the elastic tissue model. A suitable value for $\alpha$ in (3) was determined empirically $(\alpha=0.001)$. Furthermore, a rigid and a free form spline-based registration without volume or elasticity penalties [13] (FFD) was performed for comparison.

Qualitative results can be seen in Fig. 2 which shows the remaining artifacts in the difference images for the different registration methods. Since accurately identifying suitable corresponding landmarks in the breast MR images is very difficult, the quantitative evaluation of the method is here given by the decrease in sum of squared differences (SSD) (Table1). Note that the presented registration 


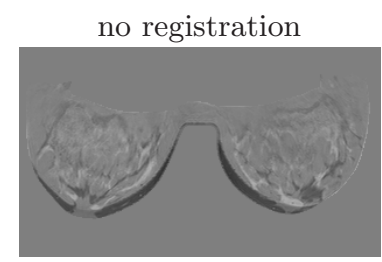

$\mathrm{MTM}+\mathrm{BC} 1$

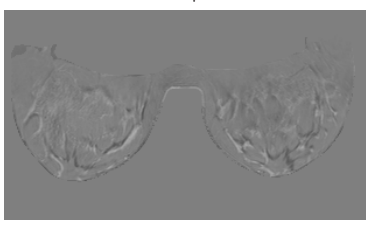

rigid

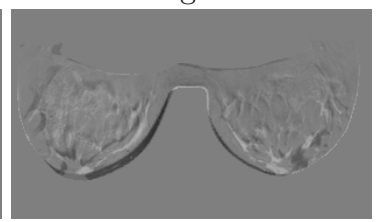

$\mathrm{MTM}+\mathrm{BC} 2$

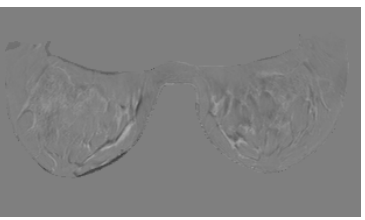

FFD

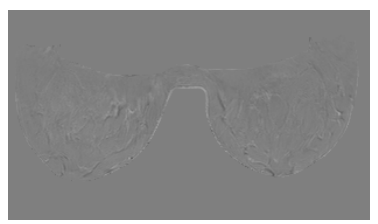

$\mathrm{MTM}+\mathrm{BC} 3$

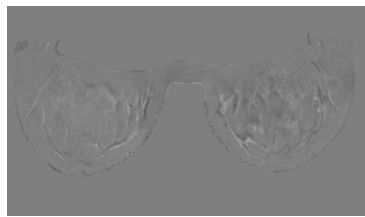

Fig. 2. Subtraction image between reference and deformed floating after different registration approaches for volunteer 1

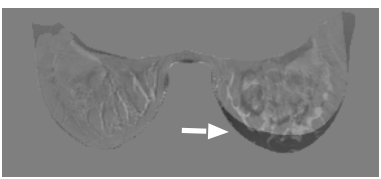

(a)

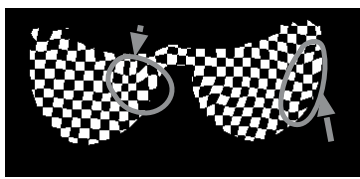

(b)

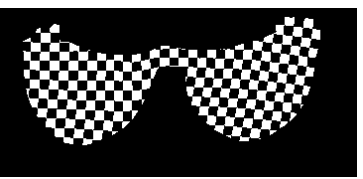

(c)

Fig. 3. (a) Subtraction image after MTM+BC2 for volunteer 3 with surface mismatch indicated. (b) and (c) Deformation field after FFD and MTM+BC3 with unplausible deformation field indicated.

Table 1. Left: remaining SSD after different registration approaches, relative to the SSD after rigid registration. Right: variation in Jacobian, characterized as minimal and maximal value and 5 th and 95 th percentile (90\% of the values lies between $p_{5}$ and $\left.p_{95}\right)$.

\begin{tabular}{|l|ccc|cccc|}
\hline & \multicolumn{3}{|c|}{ Remaining SSD } & \multicolumn{4}{|c|}{ Jacobian (averaged over volunteers) } \\
& volunteer & 1 volunteer & 2 volunteer & min & $p_{5}$ & $p_{95}$ & $\max$ \\
\hline BC 1 & $21 \%$ & $41 \%$ & $19 \%$ & 0.87 & 0.98 & 1.03 & 1.16 \\
BC 2 & $18 \%$ & $35 \%$ & $28 \%$ & 0.87 & 0.98 & 1.03 & 1.16 \\
BC 3 & $15 \%$ & $28 \%$ & $15 \%$ & 0.80 & 0.98 & 1.06 & 1.22 \\
FFD & $9 \%$ & $25 \%$ & $9 \%$ & 0.02 & 0.40 & 1.54 & 4.29 \\
\hline
\end{tabular}

method, in contrast to the FFD method, does not minimizes the SSD explicitly (BC1) or does so only around the boundaries (BC2 and BC3). The limited reduction in SSD for the registration of volunteer 3 with $\mathrm{BC} 2$ is caused by a clear misregistration of the skin surfaces, as illustrated in Fig. 3(a).

Application of the three boundary conditions on the patient data reduces the SSD on average to $45 \pm 16 \%, 43 \pm 14 \%$ and $38 \pm 14 \%$ of the rigid SSD for $\mathrm{BC} 1$, $\mathrm{BC} 2$ and $\mathrm{BC} 3$ respectively. FFD registration results in a reduction to $19 \pm 8 \%$. 
Biomechanical plausibility of the deformation field is assessed by measuring the local volume change as the Jacobian of the deformation field (Table1), which should be around 1 for an almost incompressible tissue. Deviations up to $20 \%$ are present in the biomechanically based registration method, but only in a very limited amount, since $90 \%$ of the Jacobians in the tissue is between 0.98 and 1.06. The largest deviations in local volume changes are located at the borders and especially in the small region between the breasts, while the Jacobian is close to 1 in the center of the breast. The FFD results in a deformation with much higher local volume changes with Jacobians between 0.02 and 4.29 (90\% between 0.40 and 1.54), as illustrated in Fig. 3. (b-c).

The introduction of more complex boundary conditions increases the computation time: our current $\mathrm{C}++$ implementation solves a problem with fixed correspondences in 20 seconds, while the BC1 scheme needs approximately 3 minutes and the BC2 and BC3 scheme about 45 minutes (2.19 GHz and $2 \mathrm{~Gb}$ RAM).

\section{Discussion and Conclusion}

We presented a biomechanically based registration method with a set of iteratively updated boundary conditions to align corresponding boundary surfaces. This makes biomechanically based registration feasible even when establishing accurate point-to-point correspondences is difficult or impossible in the initialization phase. Three schemes to update the boundary conditions are presented: the first is based on sliding surface contact, the second on local image intensity alignment and the third on the combination of surface attraction and local intensities. Application of the first scheme preserved mismatches at the surfaces due to segmentation inaccuracies. The second and third scheme require the determination of a weighting parameter $\alpha$, which was empirically determined to be consistent over the set of volunteer data. The second scheme was found to be more sensitive to $\alpha$ than the third one. The second scheme produced in some cases large mismatches when the image based force could not resist the internal forces in the elastic image, depending on the choice of $\alpha$. The third scheme resulted in the best alignment of the images in terms of SSD, combining successfully the initial segmentation of the surface with a refinement based on image intensities.

The reduction in SSD is higher for the volunteer images than for the clinical data. We hypothesize this to be due to the anatomical differences between the clinical images, but this needs to be investigated in a more in-depth clinical validation study. Analysis of the Jacobian of the deformation field shows that the three schemes result in physically plausible tissue deformations. This contrasts with FFD registration, which obtained a lower SSD, but at the expense of unrealistically high local volume changes in the whole image volume.

The advantage of the proposed method is the inherent compliance with the physical behavior of the tissue, without the need for extra conditions or penalties. The deformation is guided by a set of sparse boundary conditions in regions with known correspondences, thus reducing the risk of overfitting. 
Future work includes the development of an automatic segmentation for the breast tissue and the thorough investigation of the influence of segmentation inaccuracies on the performance of the three boundary condition schemes. A more efficient implementation of the second and third scheme could result in an important reduction of computation time. Moreover, we plan to adapt the parameter $\alpha$ automatically to the local Jacobian or internal elastic forces to provide maximal flexibility while preserving physically realistic deformations.

\section{References}

1. Ferrant, M., Nabavi, A., Macq, B., Jolesz, F., Kikinis, R., Warfield, S.: Registration of 3-D intraoperative MR images of the brain using a finite-element biomechanical model. IEEE Transactions on Medical Imaging 20, 1384-1397 (2001)

2. Clatz, O., Delingette, H., Talos, I.F., Golby, A., Kikinis, R., Jolesz, F., Ayache, N., Warfield, S.: Robust non-rigid registration to capture brain shift from intraoperative MRI. IEEE Transactions on Medical Imaging 24, 1417-1427 (2005)

3. Crouch, J.R., Pizer, S.M., Chaney, E.L., Hu, Y.C., Mageras, G.S., Zaider, M.: Automated finite-element analysis for deformable registration of prostate images. IEEE Transactions on Medical Imaging 26, 1379-1390 (2007)

4. Brock, K.K., Sharpe, M.B., Dawson, L.A., Kim, S.M., Jaffray, D.A.: Accuracy of finite element model-based multi-organ deformable image registration. Medical Physics 32, 1647-1659 (2005)

5. Zienkiewicz, O.C., Taylor, R.L.: The finite element method. McGraw-Hill, London (1988)

6. Liang, J., Yan, D.: Reducing uncertainties in volumetric image based deformable organ registration. Medical Physics 30, 2116-2122 (2003)

7. Roose, L., Mollemans, W., Loeckx, D., Maes, F., Suetens, P.: Biomechanically based elastic breast registration using mass tensor simulation. In: Medical Image Computing and Computer Assisted Intervention (2006)

8. Carter, T., Sermesant, M., Cash, D., Barratt, D., Tanner, C., Hawkes, D.: Application of soft tissue modelling to image-guided surgery. Medical Phyiscs \& Engineering 27(10), 893-909 (2005)

9. Cotin, S., Delingette, H., Ayache, N.: A hybrid elastic model allowing real-time cutting, deformations and force-feedback for surgery training and simulation. The Visual Computer 16, 437-452 (2000)

10. Tanner, C., Schnabel, J.A., Hill, D.L.G., Hawkes, D.J., Leach, M.O., Hose, D.R.: Factors influencing the accuracy of biomechanical breast models. Medical Physics 33, 1758-1769 (2006)

11. Ruiter, N., Muller, T., Stotzka, R., Gemmeke, H., Reichenbach, J., Kaiser, W.: Automatic image matching for breast cancer diagnostics by a 3D deformation model of the mamma. Biomed. Tech. 47, 644-647 (2002)

12. Mollemans, W., Schutyser, F., Nadjmi, N., Maes, F., Suetens, P.: Predicting soft tissue deformations for a maxillofacial surgery planning system: from computational strategies to a complete clinical validation. Medical Image Analysis 11, 282-301 (2007)

13. Loeckx, D.: Automated nonrigid intra-patient image registration using B-splines. $\mathrm{PhD}$ thesis, Katholieke Universiteit Leuven (2006) 